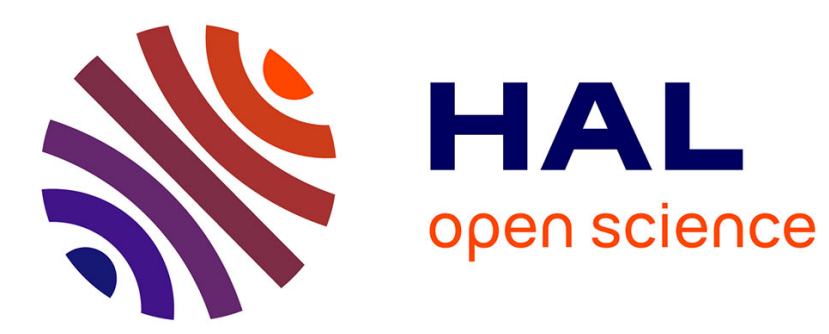

\title{
Polymer melt : reptation of a chain and viscosity
}

J. Des Cloizeaux

\section{To cite this version:}

J. Des Cloizeaux. Polymer melt: reptation of a chain and viscosity. Journal de Physique Lettres, 1984, 45 (1), pp.17-26. 10.1051/jphyslet:0198400450101700 . jpa-00232302

\section{HAL Id: jpa-00232302 https://hal.science/jpa-00232302}

Submitted on 1 Jan 1984

HAL is a multi-disciplinary open access archive for the deposit and dissemination of scientific research documents, whether they are published or not. The documents may come from teaching and research institutions in France or abroad, or from public or private research centers.
L'archive ouverte pluridisciplinaire HAL, est destinée au dépôt et à la diffusion de documents scientifiques de niveau recherche, publiés ou non, émanant des établissements d'enseignement et de recherche français ou étrangers, des laboratoires publics ou privés. 
Classification

Physics Abstracts

$05.40-46.30 \mathrm{~J}-81.20 \mathrm{~S}$

\title{
Polymer melt : reptation of a chain and viscosity
}

\author{
J. des Cloizeaux
}

Service de Physique Théorique, CEN Saclay, Orme des Merisiers, 91191 Gif-sur-Yvette Cedex, France

(Reçu le 8 juillet 1983, accepté le 10 novembre 1983)

\begin{abstract}
Résumé. - La viscosité d'un fondu polymère est calculée en supposant que la relaxation découle de la reptation d'une chaîne polymère élastique dans un tube mince. Le modèle mathématique utilisé est semblable à un modèle proposé par Doi et il est étudié de manière très détaillée. Malheureusement, il apparaît, que les résultats expérimentaux ne peuvent être reproduits de cette façon et qu'il faut trouver une approche plus réaliste.
\end{abstract}

\begin{abstract}
The viscosity of a polymer melt is calculated by assuming that the relaxation process results from the reptation of an elastic polymer in a thin tube. The mathematical model used here is similar to a model proposed by Doi, and is treated in great detail. Unfortunately, it appears that the experimental results cannot be reproduced in this way and that a more realistic approach is needed.
\end{abstract}

\section{Introduction.}

The experimental curve representing on a log-log plot the dependence of the viscosity $\eta$ of a polymer melt with respect to the number of monomers $N$ appears as made [1] of two straight lines with slopes 1 and 3.4 (see Fig. 1). For large $N$, the current reptation theories $[2,3]$ predict that the value of the slope should be 3. Graessley [4] has suggested that the anomaly could be explained by crossover effects. M. Doi [5] has claimed that the experimental result can be reproduced, for $N \gg 1$, by representing each polymer as an elastic string reptating in a thin tube. Here, this question is reexamined and we study in great detail a model which is similar to the Doi model.

\section{The model.}

The polymer is represented by an elastic string made of $N$ links (see Fig. 2) moving along an axis which represents the tube; however the tube itself is considered as a Brownian curve (see Fig. 3). The lengths of the links are independent random variables.

The probability distribution of the length of a link is assumed to be

$$
P_{1}(l)=\frac{1}{a} \mathrm{e}^{-l / a}, \quad l>0 .
$$




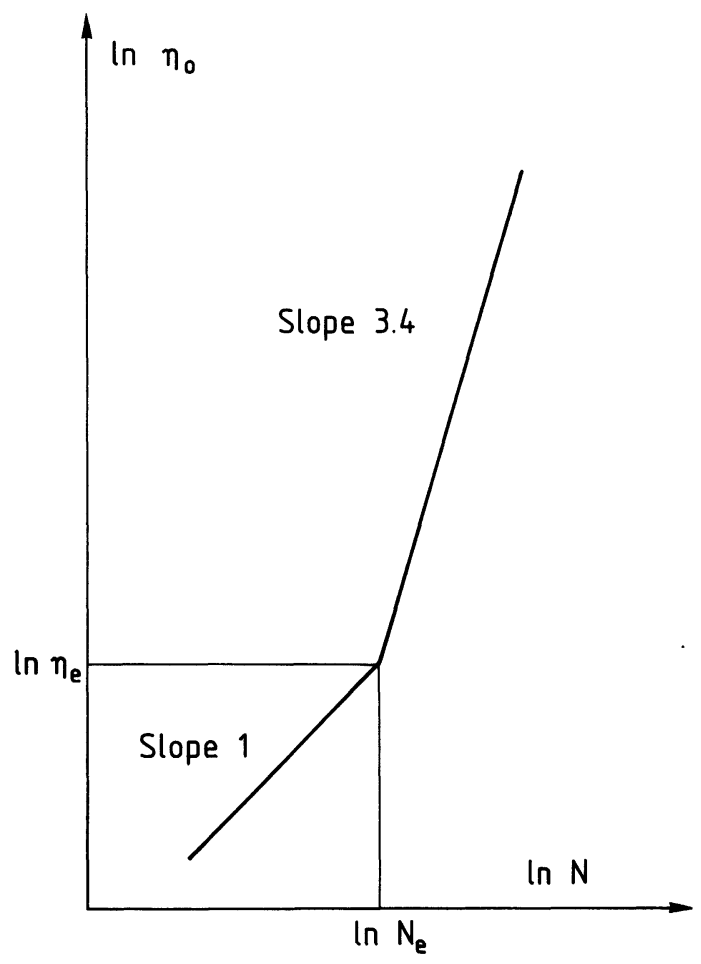

Fig. 1. - Log-log plot of $\eta$ versus $N$ : schematic representation of experimental results.

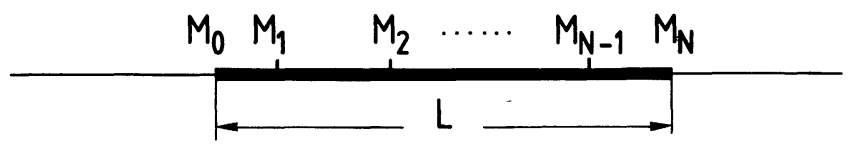

Fig. 2. - A representation of the polymer on an axis which represents the tube. The polymer is elastic and diffuses along the axis. The points $\mathbf{M}_{j}$ are the extremities of the links. Note that two points never cross each other.

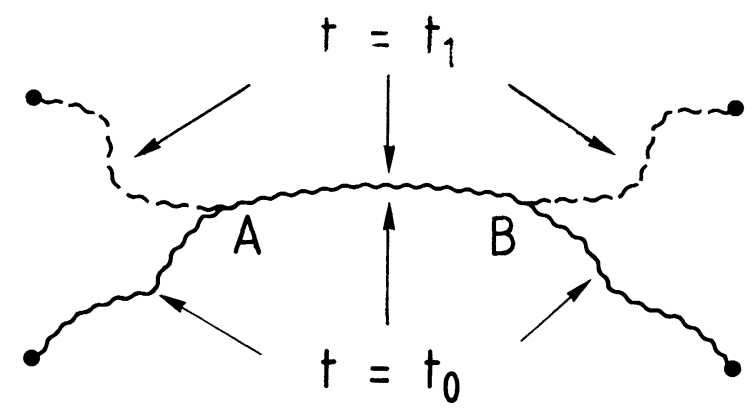

Fig. 3. - The tube (or polymer) at time $t=t_{0}$ and at time $t=t$. Between A and B, we see the conserved part of the tube. 
The probability distribution for the end to end distance is

$$
P_{N}(L)=\frac{L^{N-1}}{a(N-1) !} \mathrm{e}^{-L / a}
$$

Thus we have $\langle L\rangle=N a\left\langle(L-\langle L\rangle)^{2}\right\rangle=N a^{2}$. Let $x$ and $y$ (with $y>x$ ) be the coordinates of the end points $M_{0}$ and $M_{n}$ on the axis.

We describe the time evolution of the probability distribution $\mathbb{P}_{N} \equiv \mathbb{P}_{N}(x, y, t)$ by using the (approximate) Fokker-Planck equation

$$
\partial_{t} \mathbb{P}=(2 D / N)\left\{\partial_{x}\left(\left[\partial_{x}+\partial_{x} V_{N}(y-x)\right] \mathbb{P}_{N}\right)+\partial_{y}\left(\left[\partial_{y}+\partial_{y} V_{N}(y-x)\right] \mathbb{P}_{N}\right)\right\}
$$

where $V_{N}(L)=-\ln P_{N}(L)$ and $D$ is a diffusion constant.

This equation can be transformed by setting

$$
\mathbb{P}(x, y, t)=\mathrm{e}^{-V_{N}(y-x) / 2} Q(x, y, t) .
$$

We obtain

$$
\partial_{t} Q_{N}=-H_{N} Q_{N}
$$

where $H_{N}$ is the positive Hermitian operator

$$
H_{N}=-\frac{2 D}{N}\left[\partial_{x}^{2}+\partial_{y}^{2}-\frac{(N-1)(N-3)}{2(y-x)^{2}}+\frac{N-1}{a(y-x)}-\frac{1}{2 a^{2}}\right] .
$$

The relaxation modes correspond to eigenfunctions $\varphi(x, y)$ of $H_{N}$; these eigenfunctions are defined in the domain $y>x$; they are solutions of a Whittaker equation [6]. The eigenvalues which correspond to the relaxation of the end-to-end distance are

$$
\omega_{N, p}=\frac{D}{N a^{2}}\left[1-\left(\frac{N-1}{N-1+2 p}\right)^{2}\right]
$$

with $p=0,1,2, \ldots$

\section{The conserved tube length $l_{N}(t)$.}

At time $t=0$, the system is supposed to be in equilibrium. Let $l_{N}(t)$ be the (average) conserved tube length at time $t$. This function can be defined by

$$
l_{N}(t)=\int_{0}^{\infty} \mathrm{d} y \int_{-\infty}^{0} \mathrm{~d} x \mathbb{P}_{\dot{N}}(x, y ; t)
$$

where $\mathbb{P}_{N}(x, y ; t)$.is that solution of equation 3 which is defined in the domain $x \leqslant 0 \leqslant y$ and which obeys the boundary conditions

$$
\begin{aligned}
& \mathbb{P}_{\dot{N}}(x, y ; 0)=P_{N}(y-x) \\
& \mathbb{P} \cdot(x, 0 ; t)=P_{\dot{N}}(0, y ; t)=0, \quad t>0
\end{aligned}
$$

(therefore $l(0)=N a$ ).

In connection with $\mathbb{P}(x, y ; t)$, we shall introduce the eigenfunctions $\varphi_{\alpha}^{\cdot}(x, y)$ of $H_{N}$ which are defined in the same domain $x<0<y$ and vanish for $x=0$ and for $y=0$. Let $\omega_{N, \alpha}$ be the 
corresponding eigenvalues. The function $l_{N}(t)$ can be written in the form

$$
l_{N}(t)=\sum_{\alpha} A_{N, \alpha} \mathrm{e}^{-\omega_{\dot{N}, \alpha} t}
$$

where $A_{N, \alpha}$ can be expressed in terms of $\varphi_{\alpha}^{\cdot}(x, y)$. The quantities $\omega_{N, \alpha}$ and $A_{N, \alpha}$ cannot be exactly calculated but various approximations can be made so as to obtain soluble Whittaker equations.

Like Doi [5], we may assume that the stress relaxation function $G(t)$ is proportional to the conserved tube length $l_{N}(t)$. Consequently, we may write :

$$
\frac{\eta}{\eta_{0}}=\frac{D}{a^{2}} \int_{0}^{\infty} \mathrm{d} t\left[l_{N}(t) / N a\right]
$$

where $\eta$ is the viscosity of the melt and $\eta_{0}$ a constant.

\section{Properties of $l_{N}(t)$.}

For $N \gg 1$ (rigid rod limit), we get (in a non trivial way) the classical result

$$
l_{N}(t)=\frac{8 N a}{\pi^{2}} \sum_{m=0}^{\infty} \frac{1}{(2 m+1)^{2}} \exp \left[-D \frac{(2 m+1)^{2} \pi^{2}}{a^{2} N^{3}} t\right]
$$

(see Fig. 4).

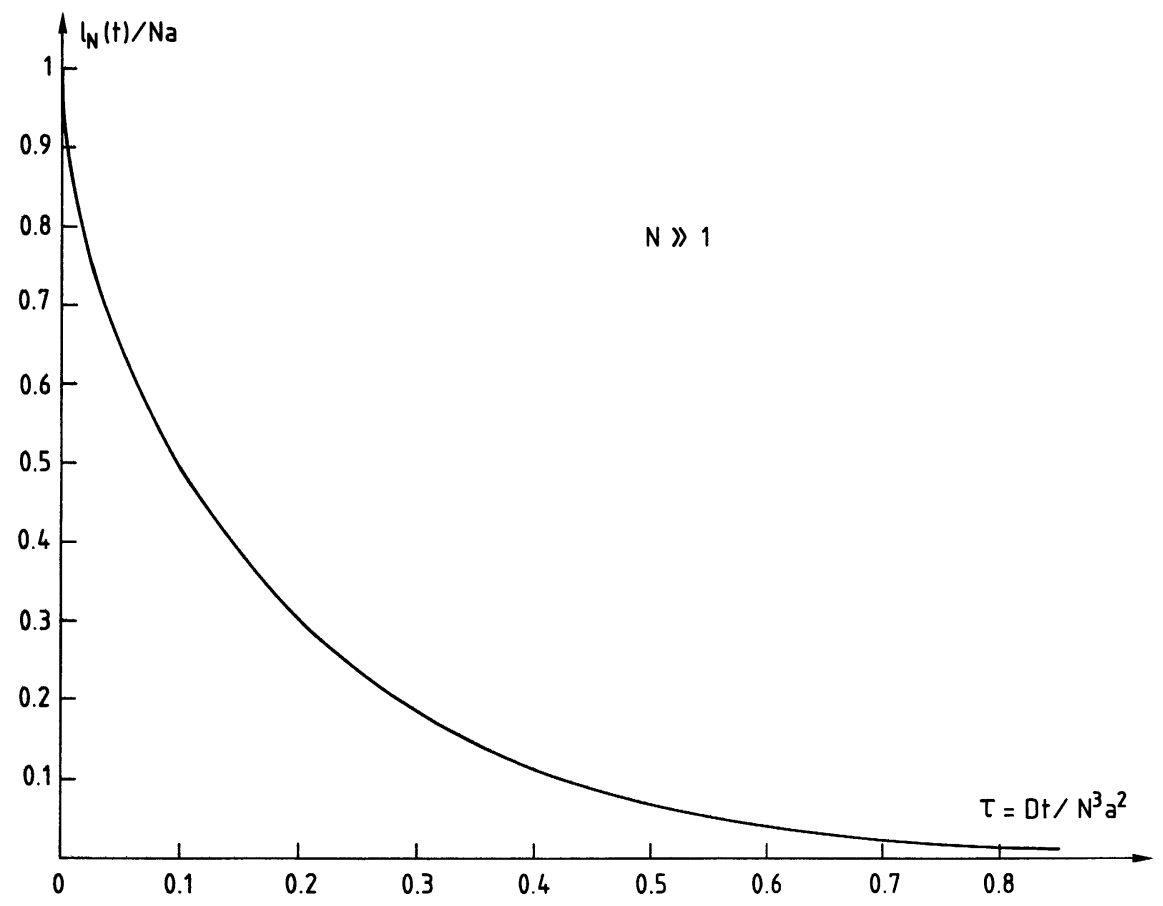

Fig. 4. - The function $l_{N}(t)$ in the large $N$ limit $(L=N a)$. 
For $N=1$, we also find an exact result

$$
l_{1}(t)=\frac{a}{2 \pi} \mathrm{e}^{-D t / a^{2}} \int_{0}^{\infty} \mathrm{d} \rho \rho^{2}(1+\rho)^{-1 / 2}(1+\rho / 2)^{-3} \mathrm{e}^{-D t \rho / 2 a^{2}}
$$

(see Fig. 5).

For small $t$ and any $N$, we can show that

$$
l_{N}(t) / N a=1-4\left(\frac{2 D t}{a^{2} N^{3} \pi}\right)^{1 / 2}+\cdots
$$

and the result is simple because in this limit only the diffusive part of equation 6 plays a rôle. Equation 14 is compatible with equation 13. On the contrary,(14) and (12), which are both exact, are not compatible; the small difference is the result of an inversion of limits.

For large $t$, in agreement with equation 10, we have

$$
l_{N}(t)=A_{N, 0} \mathrm{e}^{-t \omega_{N}, 0}
$$

where $\omega_{N, 0}$ is the smallest eigenvalue.

It is convenient to set :

$$
\begin{aligned}
\omega_{N, 0} & =\frac{D}{a^{2} N^{3}} \Omega_{N} \\
A_{N, 0} & =\frac{8 a N}{\pi^{2}} h_{N}
\end{aligned}
$$

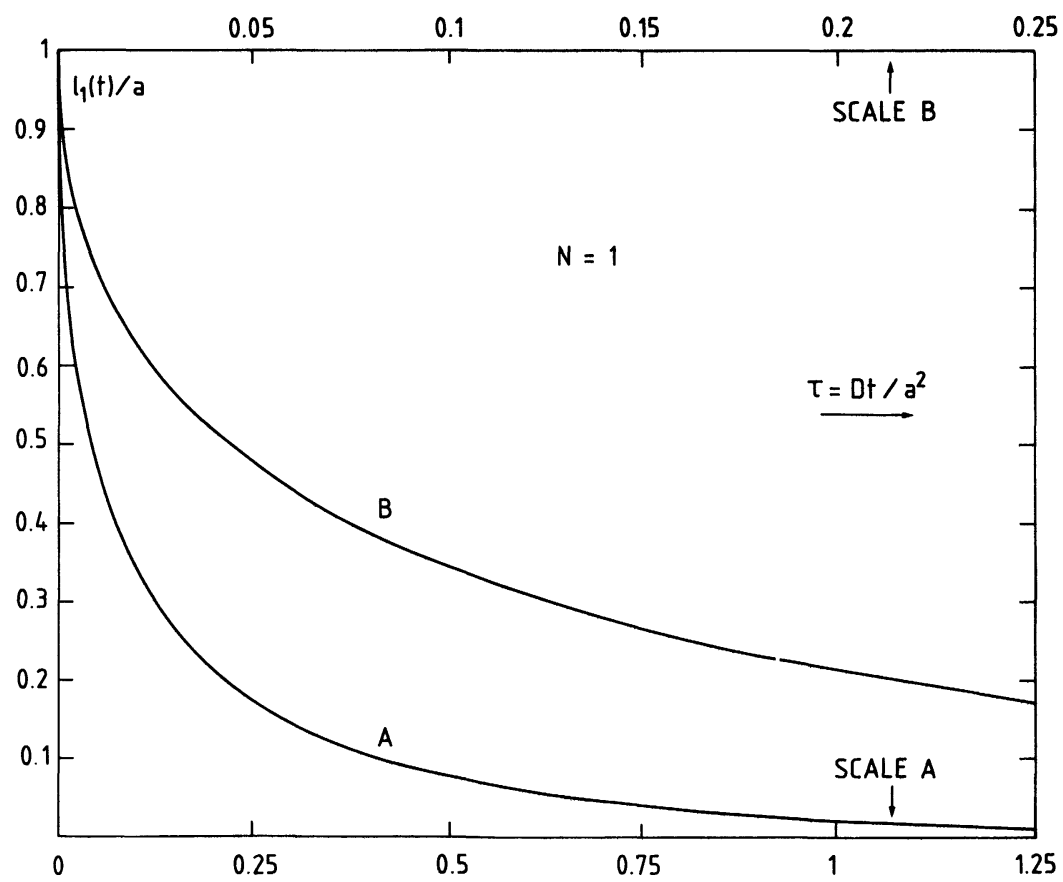

Fig. 5. - The function $l_{1}(t)(L=a)$. Both curves correspond to the same function. 
and we have found exact bounds for $\Omega_{N}$ :

$$
\Omega_{N}^{\prime}<\Omega_{N}<\Omega_{N}^{\prime \prime}
$$
$\Omega_{N}^{\prime \prime}$ has been obtained by using a trial function of the form $\cos \left[\frac{\pi}{2}\left(\frac{y+x}{y-x}\right)\right] \varphi(y-x)$ and is given
by

$$
\begin{aligned}
& \Omega_{N}^{\prime \prime}=N^{2}\left[1-\left(\frac{N-1}{N^{\prime \prime}}\right)^{2}\right] \\
& N^{\prime \prime}=1+\left[(N-2)^{2}+4 \pi^{2} / 3\right]^{1 / 2}
\end{aligned}
$$

$\Omega_{N}^{\prime}$ has been obtained by a rather similar variational method and is given by :

$$
\begin{aligned}
& \Omega_{N}^{\prime}=N^{2}\left[1-\left(\frac{N-1}{N^{\prime}}\right)^{2}\right] \\
& N^{\prime}=1+\left[(N-2)^{2}+\pi^{2}\right]^{1 / 2}
\end{aligned}
$$

(see Fig. 6).

Moreover, $\Omega_{1}^{\prime}=\Omega_{1}$ and $\Omega_{N}^{\prime} \simeq \Omega_{N}$ for $N \gg 1$. Thus, $\Omega_{N}^{\prime}$ can be considered as a good approximation for $\Omega_{N}$.

A reasonable approximation has also been found for $h_{N}$ namely

$$
h_{N}^{\prime}=2 \mathrm{e}^{3}(N-1)^{N^{\prime}+2}(N+2)^{-N-1 / 2}\left(N^{\prime}\right)^{N-N^{\prime}-1 / 2}\left(N^{\prime}+N-1\right)^{-1}
$$

(see Fig. 7).

Now, all these results can be combined to construct an approximate expression of $l_{N}(t)$ for all values of $N$ and $t$.

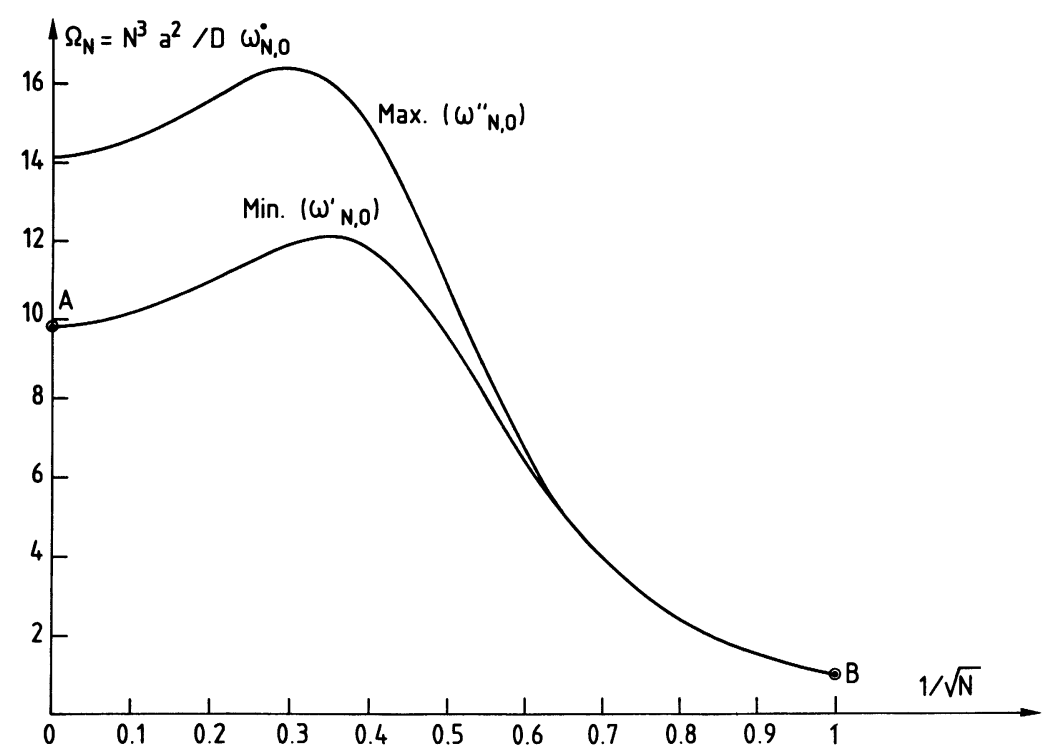

Fig. 6. - Dependence of $\omega_{N, 0}$ with respect to $N$. We have $\omega_{N, 0}^{\prime}<\omega_{N, 0}<\omega_{N, 0}^{\prime \prime}$ and the curves represent the upper bound $\omega_{N, 0}^{\prime \prime}$ and the lower bound $\omega_{N, 0}^{\prime}$. The points A and B are exact. 


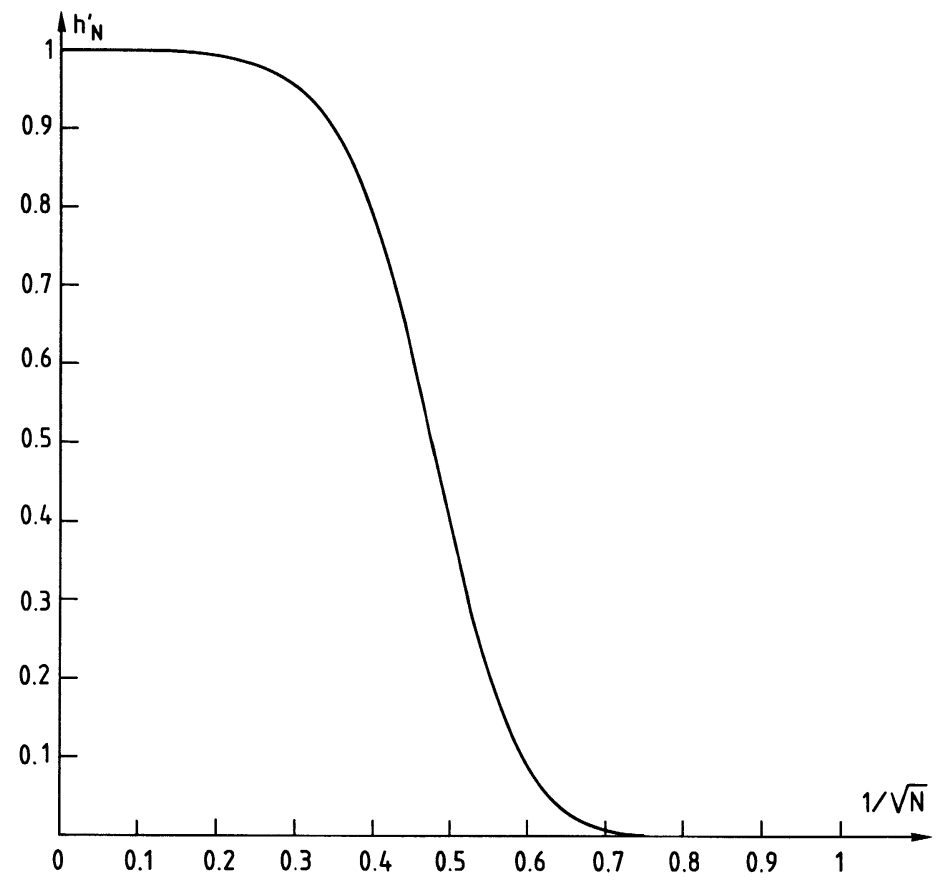

Fig. 7. - The coefficient $h_{N}^{\prime}$ versus $N^{-1 / 2}$.

Setting $\tau=D t / N^{3} a^{2}$, we write

$$
l_{N}(t) / N a=\mathrm{e}^{-\Omega_{N}^{\prime} \tau}\left[1-\beta_{N}+\frac{\beta_{N}}{2 \pi} \int_{0}^{\infty} \mathrm{d} \rho \rho^{2}(1+\rho)^{-1 / 2}(1+\rho / 2)^{-3} \mathrm{e}^{-\rho \tau / 2 \beta_{N}^{2}}\right]
$$

with

$$
\beta_{N}=1-8 \pi^{-2} h_{N}^{\prime}
$$

This expression coincides with equation 14 for $t$ small, and with equation 13 for $N=1$; it is nearly equal to the result of equation 12 for $N \gg 1$ and for $t$ large it gives a good approximation of equation 15 .

\section{Calculation of $\boldsymbol{\eta}_{\boldsymbol{N}}$.}

Combining (11) and (19), we find

$$
\eta_{N} / \eta_{0}=N^{3}\left[\left(1-\beta_{N}\right) \frac{1}{\Omega_{N}^{\prime}}+\frac{\beta_{N}}{2 \pi} \int_{0}^{\infty} \mathrm{d} \rho \rho^{2}(1+\rho)^{-1 / 2}(1+\rho / 2)^{-3}\left(\Omega_{N}^{\prime}+\rho / 2 \beta_{N}^{2}\right)^{-1}\right] .
$$

Finally, we may set

$$
\xi_{N}=2 \Omega_{N}^{\prime} \beta_{N}=2 \Omega_{N}^{\prime}\left[1-8 \pi^{-2} h_{N}^{\prime}\right]^{2}
$$

and a straightforward calculation shows that

$$
\eta_{N} / \eta_{0}=2 N^{3}\left[\left(\beta_{N}^{2} / \xi_{N}\right)+\beta_{N}^{3} F\left(\xi_{N}\right)\right]
$$


where

$$
F(\xi)=-\frac{1}{\xi}+\frac{8}{\pi(2-\xi)^{2}}-\frac{4+\xi^{2}}{(2-\xi)^{3}}+\frac{8}{\pi} \frac{\xi^{2}}{(2-\xi)^{3}} f(\xi)
$$

and

$$
\begin{array}{rlrl}
f(\xi) & =\frac{1}{(\xi-1)^{1 / 2}} \operatorname{Arctg}(\xi-1)^{1 / 2} & \xi>1 \\
& =\frac{1}{(1-\xi)^{1 / 2}} \ln \left[\frac{1+(1-\xi)^{1 / 2}}{\xi^{1 / 2}}\right] \quad \xi<1 .
\end{array}
$$

The result is shown on figures 8 and 9.

\section{Discussion and conclusion.}

The elasticity which is attributed to the polymer string produces effects which go in the right direction ; thus, we see on the log-log plot of figure 8 that the slope of the curve can be larger than 3 and the effect appears even more clearly on figure 9 .

Still, the result of this careful investigation is rather disappointing. Our model is unable to produce the large effects which are needed to explain the experimental results. In particular, the so-called " Rouse region » is very badly represented, appreciable effects occur only for rather

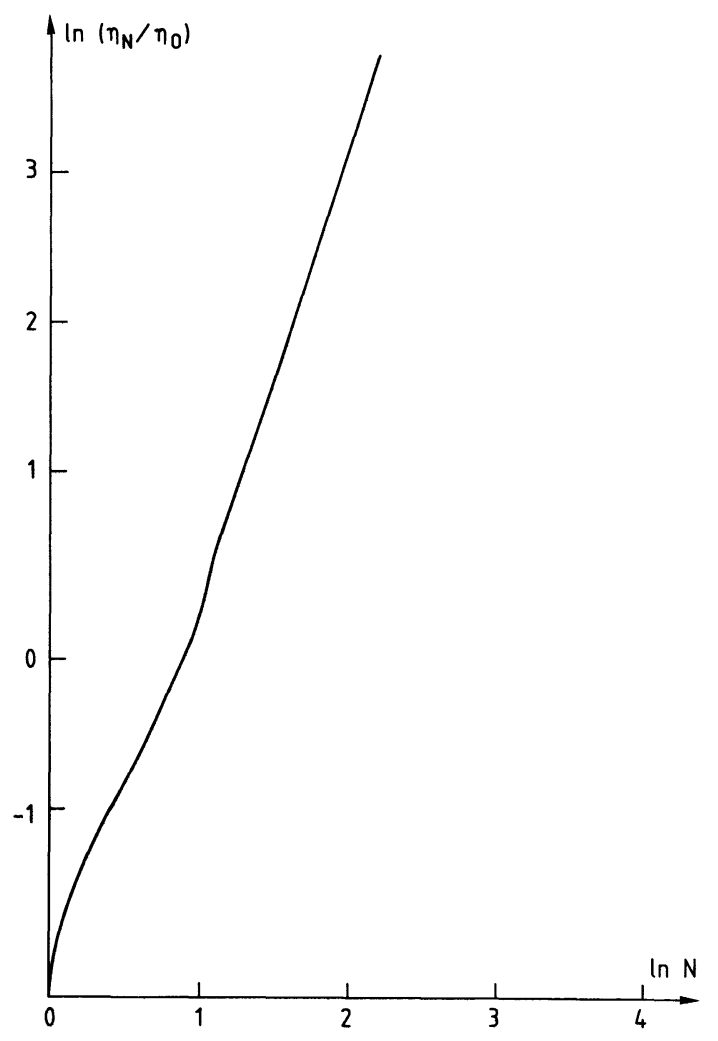

Fig. 8. - Log-log plot of $\eta / \eta_{0}$ versus $N$ (given by the calculation). 


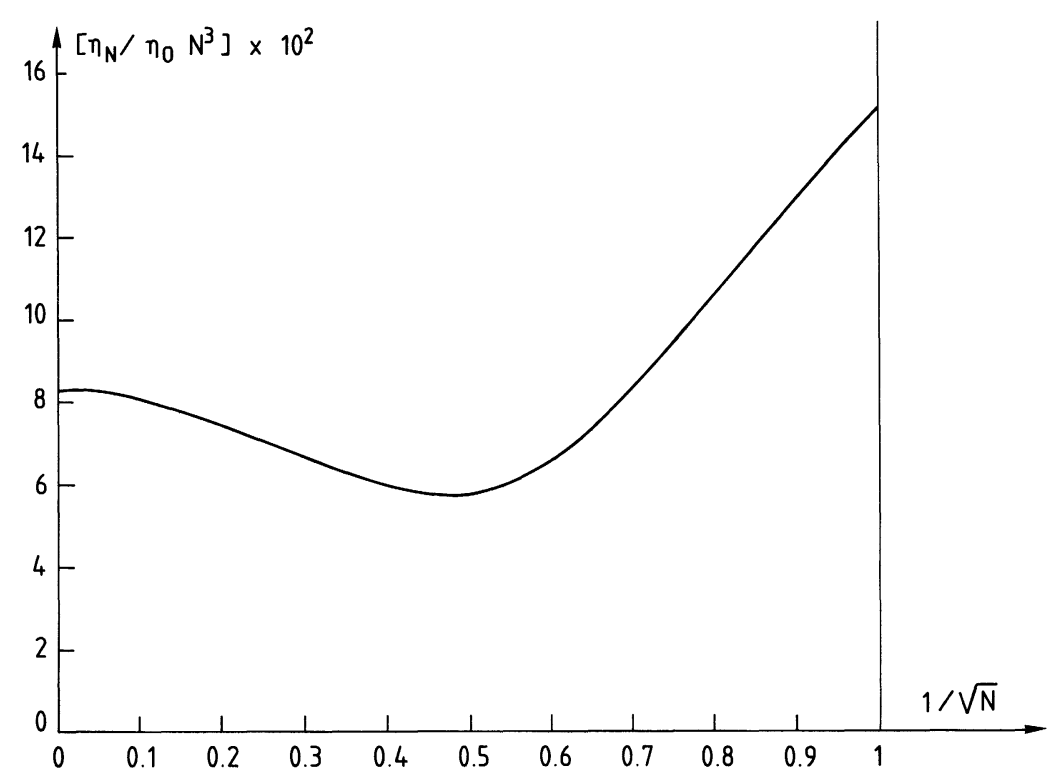

Fig. 9. - Another representation of the dependence of $\eta / \eta_{0}$ with respect to $N$ (given by the calculation).

small values of $N(N<3.5)$ and, for $N \gg 1$, the slope is only 3. It also appears that small modifications of the model cannot change the results in a drastic way and, in particular, Doi's model [5] should not be better than ours.

Actually, we see on figure 6 that $\Omega_{N} / \Omega_{N}^{\prime}<1.5$ for all $N$ and moreover $\lim _{N \rightarrow \infty} \Omega_{N} / \Omega_{N}^{\prime}=1$. Thus, as $\eta$ is fundamentally proportional to $1 / \Omega_{N}$, our result differs from the exact answer at most by a factor 1.5 for all $N$, and probably by a smaller factor in the regions of interest. On the other hand, neglecting the higher modes (see Eq. 3) may lead to an error of the same magnitude. However, the experimental results listed by Berry and Fox [1] show that straight lines of slope 3.4 are observed at least over two decades and, with respect to lines of slope 3, two decades correspond to a factor $10^{0.8}=6.3$. Moreover if Graessley's interpretation [4] is correct the real factor should be substantially larger because the effective index cannot pass abruptly from 3.4 to 3 .

Thus, we may say that the viscosity properties of a polymer melt cannot be explained by the simple reptation of an elastic string in a thin tube, and, incidentally, this conclusion seems in agreement with the result of recent computer simulations [7].

Obviously, the theory has to be seriously modified and the following possibilities cannot be excluded :

1) the assumption that the stress relaxation tensor $G(t)$ is proportional to the conserved tube length $l_{N}(t)$ is not realistic,

2) sideways motions of the polymers have to be taken into account when the number of links is not large. 


\section{References}

[1] Berry, G. C. and Fox, T. G., Adv. Polym. Sci. 5 (1968) 261.

[2] De Gennes, P. G., J. Chem. Phys. 55 (1971) 572.

[3] DoI, M. and Edwards, S. F., J. Chem. Soc. Faraday Trans. II 74 (1978) 1789, 1802, 1818.

[4] Graessley, W. W., J. Polym. Sci. Polym. Phys. Ed. 18 (1980) 27.

[5] DoI, M., J. Polym. Sci. Lett. 19 (1981) 265.

DoI, M., J. Polym. Sci. Polym. Phys. Ed. 21 (1983) 667.

[6] Higher transcendental functions. Vol. 1 Bateman Manuscript Project (Mc Graw Hill) 1953, p. 248. MesSIAH, A., Mécanique Quantique I (Dunod) 1959, p. 352.

[7] Edwards, S. F., Private communication. 